\title{
ARCHÄOZOOLOGISCHE TABELLEN
}

Sabine Deschler-Erb

Anhang zur Publikation : Lawrence Andy, Religion in Vindonissa (2018), Brugg.

\begin{tabular}{|c|c|c|c|c|c|c|c|c|c|c|c|c|c|c|c|c|c|c|c|c|c|c|c|c|c|c|c|c|c|c|c|c|c|c|c|c|c|c|c|}
\hline \multirow{2}{*}{$\begin{array}{l}\text { Strukturen Phase } 1 \\
\text { Fragmentzahlen }\end{array}$} & \multicolumn{6}{|c|}{ Bachbett } & \multicolumn{5}{|c|}{$\begin{array}{l}\text { Breiter Graben westlich } \\
\text { Tempel }\end{array}$} & \multicolumn{3}{|c|}{$\begin{array}{l}\text { schmaler } \\
\text { Graben westl. } \\
\text { Tempel }\end{array}$} & \multicolumn{3}{|c|}{$\begin{array}{l}\text { Umfassungs- } \\
\text { gräben Tempel }\end{array}$} & \multicolumn{6}{|c|}{ Schichten bei cella } & \multicolumn{9}{|c|}{ Pfostenreihen } & \multicolumn{7}{|c|}{ Gruben } \\
\hline & $\overline{\dot{5}}$ & $\stackrel{2}{\stackrel{2}{\infty}}$ & $\overline{\tilde{I}}$ & $\stackrel{\sim}{\tilde{\Sigma}}$ & E & 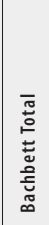 & 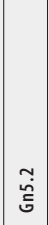 & m. & 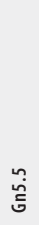 & 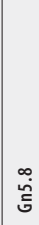 & 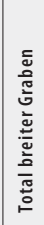 & 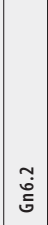 & 恣 & 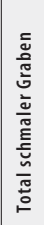 & डે & $\stackrel{\stackrel{n}{n}}{\stackrel{n}{5}}$ & 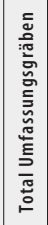 & $\stackrel{\circ}{\stackrel{\Xi}{\check{L}}}$ & $\stackrel{m}{\tilde{\Xi}}$ & $\stackrel{N}{\Xi}$ & 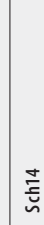 & 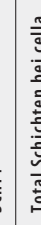 & & $\stackrel{n}{\Xi}$ & $\underset{\dot{\Xi}}{\bar{\vdots}}$ & $\overline{\underline{I}}$ & 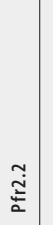 & 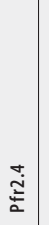 & $\stackrel{\dddot{m}}{\stackrel{2}{2}}$ & $\stackrel{m}{\stackrel{m}{ \pm}}$ & $\stackrel{n}{\stackrel{n}{2}}$ & 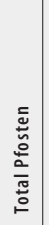 & $\overline{5}$ & 5 & కి & $\stackrel{\infty}{0}$ & $\stackrel{n}{\xi}$ & $\frac{1}{2}$ & 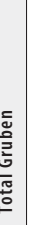 \\
\hline Bos taurus & 42 & 43 & 17 & 3 & 32 & 137 & 9 & & 2 & 45 & 56 & 16 & 11 & 27 & 23 & & 23 & 75 & 4 & 5 & & $\begin{array}{ll}9 & 9\end{array}$ & 93 & 1 & 2 & & & 1 & 1 & 2 & 5 & 12 & & & & 2 & & & 2 \\
\hline Ovis aries & & & & & & 0 & & & & & 0 & & & 0 & 1 & & 1 & & & & & & 0 & & & & & & & & & 0 & & & & & & & 0 \\
\hline Caprahircus & & & & & & 0 & & & & & 0 & & & 0 & & & 0 & & & & & & 0 & & & & & & & & & 0 & & & & & & & 0 \\
\hline Ovis a./Caprah. & 1 & 14 & 1 & & 8 & 24 & & & & & 0 & 4 & 2 & 6 & 33 & & 33 & 107 & 5 & 16 & $6 \mid 16$ & \begin{tabular}{l|l}
16 & 14
\end{tabular} & 44 & 3 & & & & & 1 & 1 & & 5 & 1 & & 1 & & 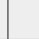 & 2 & 4 \\
\hline Sus domesticus & 2 & 12 & 14 & 15 & 9 & 52 & & 2 & & 2 & 4 & 3 & 13 & 16 & 29 & 1 & 30 & 107 & 7 & 9 & \begin{tabular}{l|l}
92 \\
9
\end{tabular} & \begin{tabular}{l|l}
2 & 14
\end{tabular} & 45 & 3 & 1 & 1 & & 2 & 2 & 2 & 1 & 12 & & 14 & 5 & 2 & 1 & 83 & 39 \\
\hline Canis familiaris & & & 5 & 6 & & 11 & & & & 1 & 1 & & & 0 & & & 0 & & & & & 1 & 1 & & & & & & & & & 0 & & & & & & & 0 \\
\hline Equus caballus & & 1 & & & & 1 & & & & & 0 & & & 0 & & & 0 & & & & & & 0 & & & & & & & & & 0 & & & & & & & 0 \\
\hline Equus sp. & 30 & 48 & 14 & 4 & & 96 & 5 & 1 & 1 & 14 & 21 & 14 & 1 & 15 & & & 0 & 2 & & & & & 2 & & & & & & & & & 0 & & & & & & & 0 \\
\hline Gallus gallus & & & & 1 & & 1 & & & & & 0 & & 1 & 1 & 4 & & 4 & 13 & & 4 & \begin{tabular}{l|l}
4 & 13 \\
\end{tabular} & 133 & 30 & & & & & 1 & 1 & & & 2 & & & & & & 4 & 4 \\
\hline Total Haustiere & 75 & 118 & 51 & 29 & 49 & 322 & 14 & 3 & 3 & 62 & 82 & 37 & 28 & 65 & 90 & 1 & 91 & 304 & 16 & 34 & \begin{tabular}{l|l}
4 & 6
\end{tabular} & \begin{tabular}{l|l}
1 & 41 \\
1
\end{tabular} & 15 & 7 & 3 & 1 & 0 & 4 & 5 & 5 & 6 & 31 & 1 & 14 & 6 & 4 & 2 & 4 & 49 \\
\hline Turdus merula & & & & & & 0 & & & & & 0 & & & 0 & & & 0 & & 0 & & & 1 & 1 & & & & & & & & & 0 & & & & & & & 0 \\
\hline Aves indet Passeriformes & & & & 1 & & 1 & & & & & 0 & & & 0 & & & 0 & & 0 & & & & 0 & & & & & & & & & 0 & & & & & & & 0 \\
\hline Rattus rattus & & & & & & 0 & & & & & 0 & & & 0 & & & 0 & 1 & 0 & & & 1 & 2 & & & & & & & & & 0 & & & & & & & 0 \\
\hline Kleinsäuger indet. & & & & & & 0 & & & & & 0 & & & 0 & & & 0 & & 0 & & & 2 & 2 & & & & & & & & & 0 & & & 1 & & & & 1 \\
\hline Rana spec & & & & & & 0 & & & & & 0 & & & 0 & & & 0 & & 0 & & & 1 & 1 & & & & & & & & & 0 & & & & & & & 0 \\
\hline Total Wildtiere & & & & 1 & & 1 & 0 & 0 & 0 & 0 & 0 & 0 & 0 & 0 & 0 & 0 & 0 & 1 & 0 & 0 & 0 & 5 & 6 & 0 & 0 & 0 & 0 & 0 & 0 & 0 & 0 & 0 & 0 & 0 & 1 & 0 & ) & 0 & 1 \\
\hline Total Haus- und Wildtiere & 75 & 118 & 51 & 30 & 49 & 323 & 14 & 3 & 3 & 62 & 82 & 37 & 28 & 65 & 90 & 1 & 91 & 305 & 16 & 34 & \begin{tabular}{l|l}
46 \\
6
\end{tabular} & \begin{tabular}{l|l}
66 & 4 \\
4
\end{tabular} & 21 & 7 & 3 & 1 & 0 & 4 & 5 & 5 & 6 & 31 & 1 & 14 & 7 & 4 & 2 & \begin{tabular}{l|l}
4 & 5
\end{tabular} & 50 \\
\hline kleine Carnivoren indet. & & & & & & 0 & & & & & 0 & & & 0 & & & 0 & 1 & & 1 & & & 2 & & & & & & & & & 0 & & & & & & & 0 \\
\hline Aves indet & & & & & & 0 & & & & & 0 & & & 0 & & & 0 & 4 & & & & 1 & 5 & & & & & & & & & 0 & & 1 & & & 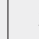 & 2 & 3 \\
\hline Total Grossgruppen & & & & & & 0 & & & & & 0 & & & 0 & & & 0 & & 0 & & & & 0 & & & & & & & & & 0 & & & & & & & 0 \\
\hline indet. Gr. Rind/Hirsch & 110 & 242 & 82 & 17 & 146 & 597 & 4 & 18 & 2 & 144 & 168 & 74 & 7 & 81 & 21 & 1 & 22 & 53 & 0 & 6 & 6 & 4 & 63 & 2 & & & & & & & & 2 & 4 & & & 17 & & \begin{tabular}{l|l}
3.2 \\
3
\end{tabular} & 24 \\
\hline indet. Gr. Schaf & & 5 & 1 & & 12 & 18 & 1 & 1 & & & 2 & 3 & 9 & 12 & 10 & & 10 & 57 & 5 & 4 & \begin{tabular}{|l|l}
420 \\
\end{tabular} & 20 & 86 & & & & & & & & & 0 & 5 & & 1 & & & \begin{tabular}{l|l}
4 \\
\end{tabular} & 10 \\
\hline indet. Gr. Schwein & & 8 & 2 & & 13 & 23 & 1 & 8 & 2 & 2 & 13 & 21 & 16 & 37 & 11 & & 11 & 56 & 11 & 7 & \begin{tabular}{|l|l}
72 \\
27
\end{tabular} & \begin{tabular}{l|l}
27 & 1
\end{tabular} & 01 & 3 & 6 & & 1 & 1 & & 4 & & 15 & 9 & 18 & 1 & 43 & 7 & $\begin{array}{lll}2 & 14\end{array}$ & 143 \\
\hline indet. Gr. Hase & & & & & & 0 & & & & & 0 & 1 & & 1 & & & 0 & 2 & 0 & & & 2 & 4 & & & & & & & & & 0 & & & & & & & 0 \\
\hline indet. & 98 & 71 & 113 & 94 & 141 & 517 & 6 & 4 & & 35 & 45 & 73 & 10 & 83 & 9 & & 9 & 11 & 18 & 8 & & & 19 & & & & & & & & & 0 & 5 & 51 & 2 & 64 & 5 & \begin{tabular}{l|l}
1 & 17
\end{tabular} & 173 \\
\hline Total Unbestimmbare & 208 & 326 & 198 & 111 & 312 & 1155 & 12 & 31 & 4 & 181 & 228 & 172 & 42 & 214 & 51 & 1 & 52 & 179 & 16 & 25 & 5 & \begin{tabular}{|l|l}
53 & 27
\end{tabular} & 73 & 5 & 6 & 0 & 1 & 1 & 0 & 4 & 0 & 17 & 23 & 69 & 4 & 124 & 13 & & 50 \\
\hline Homo sapiens & & & & & & 0 & & & & & 0 & & & 0 & & & 0 & & 0 & & & & 0 & & & & & & & & & 0 & & 36 & & & & & 36 \\
\hline Gesamtergebnis & 283 & \begin{tabular}{|l|}
444 \\
\end{tabular} & 249 & 141 & 361 & 1478 & 26 & 34 & 7 & 243 & 310 & 209 & 70 & 279 & 141 & 2 & 143 & 489 & 32 & 60 & $12 C$ & \begin{tabular}{l|l}
20 & 7
\end{tabular} & 01 & 12 & 9 & 1 & 1 & 5 & 5 & 9 & 6 & 48 & 24 & 120 & 11 & 128 & 15 & & 139 \\
\hline
\end{tabular}

Tab. 11 Brugg-Kabelwerke 2007-2008 (Bru.007.3). Tierartentabelle (n), Strukturen Phase 1. 


\begin{tabular}{|c|c|c|c|c|c|c|c|c|c|c|c|c|c|c|c|c|c|c|c|c|c|c|c|c|c|}
\hline \multirow{2}{*}{$\begin{array}{l}\text { Strukturen Phase } 1 \\
\text { Gewicht (g) }\end{array}$} & \multicolumn{6}{|c|}{ Bachbett } & \multicolumn{5}{|c|}{ Breiter Graben westlich Tempel } & \multicolumn{3}{|c|}{$\begin{array}{l}\text { schmaler Graben } \\
\text { westl.Tempel }\end{array}$} & \multicolumn{3}{|c|}{$\begin{array}{l}\text { Umfassungsgräben } \\
\text { Tempel }\end{array}$} & \multicolumn{5}{|c|}{ Schichten bei cella } & \multicolumn{3}{|c|}{ Pfostenreihen } \\
\hline & $\bar{\Xi}$ & $\stackrel{1}{\frac{5}{\infty}}$ & $\overline{\bar{I}}$ & 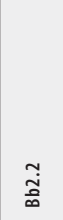 & E & 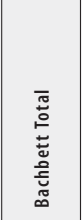 & Еี & 艿 & 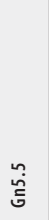 & 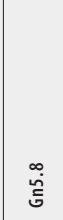 & 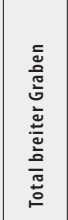 & 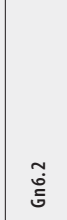 & 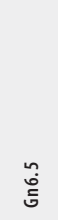 & 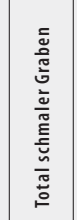 & Eิ & 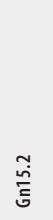 & 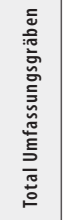 & $\stackrel{0}{\check{E}}$ & $\stackrel{m}{\tilde{n}}$ & $\stackrel{2}{\Xi}$ & $\stackrel{\frac{\sigma}{5}}{\stackrel{5}{n}}$ & 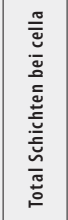 & $\stackrel{n}{\vdots}$ & 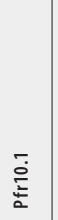 & $\overline{\bar{Z}}$ \\
\hline Bostaurus & 1380,9 & 1168 & 754,2 & 312,6 & 552,8 & 4168,5 & 199,3 & & 63,1 & 1457,1 & 1719,5 & 337 & 353 & 690 & 342,4 & & 342,4 & 797,3 & 69,2 & 51,4 & 116,8 & 1034,7 & 17,4 & 5,2 & \\
\hline Ovis aries & & & & & & 0 & & & & & 0 & & & 0 & 0,8 & & 0,8 & & & & & 0 & & & \\
\hline Ovis a./Capra h. & 4,1 & 48,9 & 4,3 & & 21,1 & 78,4 & & & & & 0 & 52,6 & 18,7 & 71,3 & 116,3 & & 116,3 & 325,8 & 33,5 & 52,4 & 53,8 & 465,5 & 17,8 & & \\
\hline Sus domesticus & 10,5 & 53,2 & 220,8 & 61,5 & 52,8 & 398,8 & & 7,3 & & 28,3 & 35,6 & 13,4 & 38,6 & 52 & 124,6 & 0,5 & 125,1 & 458 & 20,9 & 28,9 & 100,5 & 608,3 & 13,5 & 0,2 & 9,2 \\
\hline Canis familiaris & & 278,2 & & & & 278,2 & & & & & 0 & & & 0 & & & 0 & & & & & 0 & & & \\
\hline Equus caballus & $\mid 1158,1$ & 1943 & 1111 & 89,8 & & 4301,9 & 182,9 & 23,4 & 17,8 & 1717,8 & $\mid 1941,9$ & 993 & 9,8 & \begin{tabular}{l|l|}
8 & 1002,8
\end{tabular} & & & 0 & 40,5 & & & & 40,5 & & & \\
\hline Equus sp. & & & 127,7 & 191,4 & & 319,1 & & & & 3,2 & 3,2 & & & 0 & & & 0 & & & & 3,7 & 3,7 & & & \\
\hline Gallus gallus & & & & 0,4 & & 0,4 & & & & & 0 & & 1,3 & 1,3 & 5,3 & & 5,3 & 12,5 & & 4 & 8,4 & 24,9 & & & \\
\hline Total Haustiere & 2553,6 & 3491,3 & 2218 & 655,7 & 626,7 & 9545,3 & 382,2 & 30,7 & 80,9 & 3206,4 & 3700,2 & 1396 & 421,4 & $4 \quad 1817,4$ & 589,4 & 0,5 & 589,9 & 1634,1 & 123,6 & 136,7 & 283,2 & 2177,6 & 48,7 & 5,4 & 9,2 \\
\hline Turdus merula & & & & & & 0 & & & & & 0 & & & 0 & & & 0 & & & & 0,1 & 0,1 & & & \\
\hline Aves indet Passeriformes & & & & 0,1 & & 0,1 & & & & & 0 & & & 0 & & & 0 & & & & & 0 & & & \\
\hline Rattus rattus & & & & & & 0 & & & & & 0 & & & 0 & & & 0 & 0,1 & & & 0,1 & 0,2 & & & \\
\hline Kleinsäuger indet. & & & & & & 0 & & & & & 0 & & & 0 & & & 0 & & & & 0,2 & 0,2 & & & \\
\hline Rana spec & & & & & & 0 & & & & & 0 & & & 0 & & & 0 & & & & 0,1 & 0,1 & & & \\
\hline Total Wildtiere & 0 & 0 & 0 & 0,1 & 0 & 0,1 & 0 & 0 & 0 & 0 & 0 & 0 & 0 & 0 & 0 & 0 & 0 & 0,1 & 0 & 0 & 0,5 & 0,6 & 0 & 0 & 0 \\
\hline Total Haus- und Wildtiere & 2553,6 & 3491,3 & 2218 & 655,8 & 626,7 & 9545,4 & 382,2 & 30,7 & 80,9 & 3206,4 & 3700,2 & 1396 & 421,4 & $4 \mid 1817,4$ & 589,4 & 0,5 & 589,9 & 1634,2 & 123,6 & 136,7 & 283,7 & 2178,2 & 48,7 & 5,4 & 9,2 \\
\hline kleine Carnivoren indet. & & & & & & 0 & & & & & 0 & & & 0 & & & 0 & 0,7 & & 0,2 & & 0,9 & & & \\
\hline Aves indet & & & & & & 0 & & & & & 0 & & & 0 & & & 0 & 0,6 & & & 0,5 & 1,1 & & & \\
\hline Total Grossgruppen & 0 & 0 & 0 & 0 & 0 & 0 & 0 & 0 & 0 & 0 & 0 & 0 & 0 & 0 & 0 & 0 & 0 & 1,3 & 0 & 0,2 & 0,5 & 2 & 0 & 0 & 0 \\
\hline indet. Gr. Rind/Hirsch & 170,9 & 354,3 & 90,2 & 24,8 & 124,6 & 764,8 & 21,9 & 29,6 & 14 & 269,4 & 334,9 & 105,5 & 6 & \begin{tabular}{l|l|}
6 & 111,5
\end{tabular} & 28,3 & 10,4 & 38,7 & 89,2 & & 6,2 & 7,3 & 102,7 & 3,6 & & \\
\hline indet. Gr. Schaf & & 4,2 & 1,7 & & 6,4 & 12,3 & 0,4 & 0,5 & & & 0,9 & 1,6 & 6,2 & 7,8 & 6,2 & & 6,2 & 46,6 & 8,9 & 1,7 & 18,1 & 75,3 & & & \\
\hline indet. Gr. Schwein & & 8,4 & 1,8 & & 11,6 & 21,8 & 1,7 & 4,1 & 2,7 & 1,7 & 10,2 & 10,9 & 11,7 & \begin{tabular}{l|l|}
7 & 22,6 \\
\end{tabular} & 14 & & 14 & 62 & 13,7 & 4,2 & 13,6 & 93,5 & 2,9 & 1,6 & \\
\hline indet. Gr. Hase & & & & & & 0 & & & & & 0 & 0,4 & & 0,4 & & & 0 & 0,7 & & & 0,7 & 1,4 & & & \\
\hline indet. & 8,2 & 25,9 & 34,6 & 209,6 & 24 & 302,3 & 0,5 & 1,5 & & 8,9 & 10,9 & 6 & 1,1 & 7,1 & 0,1 & & 0,1 & 1,9 & & 0,5 & & 2,4 & & & \\
\hline Total Unbestimmbare & 179,1 & 392,8 & 128,3 & 234,4 & 166,6 & 1101,2 & 24,5 & 35,7 & 16,7 & 280 & 356,9 & 124,4 & 25 & \begin{tabular}{|l|l|}
5 & 149,4
\end{tabular} & 48,6 & 10,4 & 59 & 200,4 & 22,6 & 12,6 & 39,7 & 275,3 & 6,5 & 1,6 & 0 \\
\hline Homo sapiens & & & & & & 0 & & & & & 0 & & & 0 & & & 0 & & & & & 0 & & & \\
\hline Gesamtergebnis & 2732,7 & 3884,1 & 2346,3 & 890,2 & 793,3 & 10646,6 & 406,7 & 66,4 & 97,6 & 3486,4 & 4057,1 & 1520,4 & 446,4 & $\begin{array}{l}4 \\
4\end{array}$ & 638 & 10,9 & 648,9 & 1835,9 & 146,2 & 149,5 & 323,9 & 2455,5 & 55,2 & 7 & 9,2 \\
\hline
\end{tabular}

Tab. 12 Brugg-Kabelwerke 2007-2008 (Bru.007.3). Tierartentabelle (g), Strukturen Phase 1. 


\begin{tabular}{|c|c|c|c|c|c|c|c|c|c|c|c|}
\hline \multirow[b]{2}{*}{ 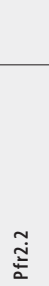 } & \multirow[b]{2}{*}{$\stackrel{+}{\stackrel{ \pm}{ \pm}}$} & \multirow[b]{2}{*}{$\underset{m}{\stackrel{m}{\rightleftarrows}}$} & \multirow[b]{2}{*}{$\underset{\stackrel{m}{\rightleftarrows}}{\stackrel{m}{2}}$} & \multirow[b]{2}{*}{ 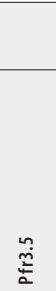 } & \multirow[b]{2}{*}{ 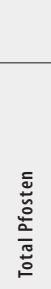 } & \multicolumn{6}{|l|}{ Gruben } \\
\hline & & & & & & $\overline{5}$ & 5 & స్ & $\stackrel{\infty}{\sigma}$ & 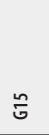 & 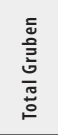 \\
\hline & 4,8 & 18,4 & 6 & 53,8 & 164,6 & & & & 5,7 & 0 & 5,7 \\
\hline & & & & & 0 & & & & & 0 & 0 \\
\hline & & 1,6 & 1, & & 21,3 & 1,1 & & 0,2 & & 4,5 & 5,8 \\
\hline & 5,3 & 15,7 & 4, & 1,7 & 50,2 & & 80,6 & 9,6 & 8,9 & 47,5 & 146,6 \\
\hline & & & & & 0 & & & & & 0 & 0 \\
\hline & & & & & 0 & & & & & 0 & 0 \\
\hline & & & & & 0 & & & & & 0 & 0 \\
\hline & 0,1 & 0,3 & & & 0,4 & & & & & 1,4 & 1,4 \\
\hline 0 & 10,2 & 36 & 71,5 & 55,5 & 236,5 & 1,1 & 80,6 & 9,8 & 14,6 & 53,4 & 159,5 \\
\hline & & & & & 0 & & & & & 0 & 0 \\
\hline & & & & & 0 & & & & & 0 & 0 \\
\hline & & & & & 0 & & & & & 0 & 0 \\
\hline & & & & & 0 & & & 0,1 & & 0 & 0,1 \\
\hline & & & & & 0 & & & & & 0 & 0 \\
\hline 0 & 0 & 0 & c & 0 & 0 & 0 & 0 & 0,1 & 0 & 0 & 0,1 \\
\hline 0 & 10,2 & 36 & 71,5 & 55,5 & 236,5 & 1,1 & 80,6 & 9,9 & 14,6 & 53,4 & 159,6 \\
\hline & & & & & 0 & & & & & 0 & 0 \\
\hline & & & & & 0 & & 0,1 & & & 0,1 & 0,2 \\
\hline 0 & 0 & 0 & c & 0 & 0 & 0 & 0,1 & 0 & 0 & 0,1 & 0,2 \\
\hline & & & & & 3,6 & 6,7 & & & 19,4 & 8 & 34,1 \\
\hline & & & & & 0 & 5,1 & & 0,3 & & 1,2 & 6,6 \\
\hline 0,7 & 1,2 & & 1,8 & & 8,2 & 7,4 & 15,9 & 0,3 & 23,7 & 25,3 & 72,6 \\
\hline & & & & & 0 & & & & & 0 & 0 \\
\hline & & & & & 0 & 0,8 & 20,9 & 0,9 & 5,8 & 6 & 34,4 \\
\hline 0,7 & 1,2 & 0 & 1,8 & 0 & 11,8 & 20 & 36,8 & 1,5 & 48,9 & 40,5 & 147,7 \\
\hline & & & & & 0 & & 111,2 & & & 0 & 111,2 \\
\hline 0,7 & 11,4 & 36 & 73,3 & 55,5 & 248,3 & 21,1 & 228,7 & 11,4 & 63,5 & 94 & 418,7 \\
\hline
\end{tabular}




\begin{tabular}{|c|c|c|c|c|c|c|c|c|c|c|c|c|c|}
\hline Strukturen Phase 2 & Gruben & & & Kapelle? & Pfostenr & ihen & & & & & & Schicht & ei cella \\
\hline Fragmentzahlen & G23 & G7 & $\begin{array}{l}\text { Total } \\
\text { Gruben }\end{array}$ & M4 & Pfr6.1 & Pfr6.2 & Pfr6.3 & Pfr6.4 & Pfr7.3 & Pfr8.1 & $\begin{array}{l}\text { Total } \\
\text { Pfosten }\end{array}$ & Sch15 & Total \\
\hline Bos taurus & 4 & 6 & 10 & 5 & 4 & 23 & 4 & 34 & & & 70 & 26 & 106 \\
\hline Ovis aries & & & 0 & & & 1 & & & & & 1 & & 1 \\
\hline Capra hircus & & & 0 & 1 & & & 1 & 1 & & & 3 & & 3 \\
\hline Ovis a./Capra h. & 6 & 5 & 11 & 14 & 8 & 14 & 1 & 45 & 4 & & 86 & 16 & 113 \\
\hline Sus domesticus & 9 & 3 & 12 & 11 & 3 & 36 & 12 & 38 & 3 & 1 & 104 & 41 & 157 \\
\hline Equus caballus & & & 0 & & & & 11 & & & & 11 & & 11 \\
\hline Equus sp. & 2 & & 2 & & & 12 & 6 & 3 & & & 21 & 2 & 25 \\
\hline Canis familiaris & & & 0 & & 1 & 2 & 5 & 1 & & & 9 & 1 & 10 \\
\hline Gallus gallus & 1 & 1 & 2 & 4 & & 4 & 1 & 7 & & & 16 & 1 & 19 \\
\hline Total Haustiere & 22 & 15 & 37 & 35 & 16 & 92 & 41 & 129 & 7 & 1 & 321 & 87 & 445 \\
\hline Rattus rattus & & & 0 & & & & & & & & 0 & 1 & 1 \\
\hline Total Wildtiere & 0 & 0 & 0 & 0 & 0 & 0 & 0 & 0 & 0 & 0 & 0 & 1 & 1 \\
\hline Total Haus- und Wildtiere & 22 & 15 & 37 & 35 & 16 & 92 & 41 & 129 & 7 & 1 & 321 & 88 & 446 \\
\hline Aves indet & & & 0 & 1 & & 1 & & 2 & & & 4 & & 4 \\
\hline Total Grossgruppen & 0 & 0 & 0 & 1 & 0 & 1 & 0 & 2 & 0 & 0 & 4 & 0 & 4 \\
\hline indet. Gr. Rind/Hirsch & 2 & 53 & 55 & 1 & 5 & 40 & 15 & 38 & 12 & & 111 & 12 & 178 \\
\hline indet. Gr. Schaf & & 4 & 4 & 3 & & 8 & & 4 & 4 & & 19 & 17 & 40 \\
\hline indet. Gr. Schwein & 17 & 4 & 21 & 28 & 3 & 11 & 16 & 15 & & & 73 & 22 & 116 \\
\hline indet. Gr. Hase & & & 0 & & & & 1 & 1 & & & 2 & & 2 \\
\hline indet. & & 3 & 3 & & 30 & 17 & & 37 & 3 & & 87 & & 90 \\
\hline Total Unbestimmbare & 19 & 64 & 83 & 32 & 38 & 76 & 32 & 95 & 19 & 0 & 292 & 51 & 426 \\
\hline Homo sapiens & & & 0 & & & & & & & & 0 & 1 & 1 \\
\hline Gesamtergebnis & 41 & 79 & 120 & 68 & 54 & 169 & 73 & 226 & 26 & 1 & 617 & 140 & 877 \\
\hline
\end{tabular}

Tab. 13 Brugg-Kabelwerke 2007-2008 (Bru.007.3). Tierartentabelle (n), Strukturen Phase 2

\begin{tabular}{|c|c|c|c|c|c|c|c|c|c|c|c|c|c|}
\hline Strukturen Phase 2 & Gruben & & & Kapelle? & Pfostenr & ihen & & & & & & Schicht b & i cella \\
\hline Gewicht (g) & G23 & G7 & $\begin{array}{l}\text { Total } \\
\text { Gruben }\end{array}$ & M4 & Pfr6.1 & Pfr6.2 & Pfr6.3 & Pfr6.4 & Pfr7.3 & Pfr8.1 & $\begin{array}{l}\text { Total } \\
\text { Pfosten }\end{array}$ & Sch15 & Total \\
\hline Bos taurus & 32 & 136,1 & 168,1 & 72,8 & 112,2 & 498,2 & 106,4 & 691,3 & & & 1408,1 & 386,9 & 2035,9 \\
\hline Ovis aries & & & 0 & & & 3,7 & & & & & 3,7 & & 3,7 \\
\hline Capra hircus & & & 0 & 0,9 & & & 4 & 4,9 & & & 8,9 & & 9,8 \\
\hline Ovis a./Capra h. & 32,9 & 19,3 & 52,2 & 27,2 & 28,1 & 68,2 & 1,7 & 126,8 & 15,2 & & 240 & 63 & 382,4 \\
\hline Sus domesticus & 29,1 & 5,9 & 35 & 22,8 & 12,6 & 165,4 & 89,6 & 183 & 24,6 & 1,9 & 477,1 & 209 & 743,9 \\
\hline Equus caballus & & & 0 & & & & 844 & & & & 844 & & 844 \\
\hline Equus sp. & 11,9 & & 11,9 & & & 188,1 & 59,6 & 51,2 & & & 298,9 & 19 & 329,8 \\
\hline Canis familiaris & & & 0 & & 0,4 & 3,2 & 46,3 & 2,6 & & & 52,5 & 3,5 & 56 \\
\hline Gallus gallus & 1,7 & 0,7 & 2,4 & 3,7 & & 1,5 & 0,5 & 5 & & & 7 & 0,9 & 14 \\
\hline Total Haustiere & 107,6 & 162 & 269,6 & 127,4 & 153,3 & 928,3 & 1152,1 & 1064,8 & 39,8 & 1,9 & 3340,2 & 682,3 & 4419,5 \\
\hline Rattus rattus & & & 0 & & & & & & & & 0 & 0,1 & 0,1 \\
\hline Total Wildtiere & 0 & 0 & 0 & 0 & 0 & 0 & 0 & 0 & 0 & 0 & 0 & 0,1 & 0,1 \\
\hline Total Haus- und Wildtiere & 107,6 & 162 & 269,6 & 127,4 & 153,3 & 928,3 & 1152,1 & 1064,8 & 39,8 & 1,9 & 3340,2 & 682,4 & 4419,6 \\
\hline Aves indet & & & 0 & 0,2 & & 0,2 & & 0,3 & & & 0,5 & & 0,7 \\
\hline Total Grossgruppen & 0 & 0 & 0 & 0,2 & 0 & 0,2 & 0 & 0,3 & 0 & 0 & 0,5 & 0 & 0,7 \\
\hline indet. Gr. Rind/Hirsch & 5,6 & 75,4 & 81 & 1,6 & 9,3 & 47,9 & 19,3 & 59,3 & 14,2 & & 150 & 38,7 & 271,3 \\
\hline indet. Gr. Schaf & & 2,8 & 2,8 & 3,8 & & 5,3 & & 1,1 & 2,8 & & 9,2 & 14,5 & 30,3 \\
\hline indet. Gr. Schwein & 11,1 & 6,5 & 17,6 & 11,4 & 6,9 & 5,7 & 4,3 & 16,7 & & & 33,6 & 25,2 & 87,8 \\
\hline indet. Gr. Hase & & & 0 & & & & 0,4 & 0,6 & & & 1 & & 1 \\
\hline indet. & & 0,9 & 0,9 & & 6,8 & 5,9 & & 6,6 & 0,4 & & 19,7 & & 20,6 \\
\hline Total Unbestimmbare & 16,7 & 85,6 & 102,3 & 16,8 & 23 & 64,8 & 24 & 84,3 & 17,4 & 0 & 213,5 & 78,4 & 411 \\
\hline Homo sapiens & & & 0 & & & & & & & & 0 & 33,8 & 33,8 \\
\hline Gesamtergebnis & 124,3 & 247,6 & 371,9 & 144,4 & 176,3 & 993,3 & 1176,1 & 1149,4 & 57,2 & 1,9 & 3554,2 & 794,6 & 4865,1 \\
\hline
\end{tabular}

Tab. 14 Brugg-Kabelwerke 2007-2008 (Bru.007.3). Tierartentabelle (g), Strukturen Phase 2. 


\begin{tabular}{|l|c|r|r|}
\hline Strukturen Phase 3 & \multicolumn{3}{l|}{ Gruben } \\
\hline Fragmentzahlen & G 16 & G 22 & Total \\
\hline Bos taurus & & 14 & 14 \\
\hline Ovis a./Capra h. & 1 & 24 & 24 \\
\hline Sus domesticus & 2 & 5 & 48 \\
\hline Gallus gallus & 3 & 90 & 7 \\
\hline Total Haustiere & & 1 & 1 \\
\hline Aves indet & 0 & 1 & 1 \\
\hline Total Grossgruppen & & 6 & 6 \\
\hline indet. Gr. Rind/Hirsch & & 21 & 21 \\
\hline indet. Gr. Schaf & & 11 & 11 \\
\hline indet. Gr. Schwein & 0 & 38 & 38 \\
\hline Total Unbestimmbare & 3 & 129 & 132 \\
\hline Gesamtergebnis & & & 17 \\
\hline
\end{tabular}

Tab. 15 Brugg-Kabelwerke 2007-2008 (Bru.007.3). Tierartentabelle (n), Strukturen Phase 3.

\begin{tabular}{l|r|r|r}
\hline Strukturen Phase 3 & \multicolumn{3}{l}{ Gruben } \\
\hline Gewicht (g) & G 16 & G 22 & \multicolumn{1}{l}{ Total } \\
\hline Bos taurus & & 140,9 & 140,9 \\
\hline Ovis a./Capra h. & & 51,1 & 51,1 \\
\hline Sus domesticus & 2,7 & 179,1 & 181,8 \\
\hline Gallus gallus & 1,7 & 3,4 & 5,1 \\
\hline Total Haustiere & 4,4 & 374,5 & 378,9 \\
\hline Aves indet & & 0,3 & 0,3 \\
\hline Total Grossgruppen & 0 & 0,3 & 0,3 \\
\hline indet. Gr. Rind/Hirsch & & 12,2 & 12,2 \\
\hline indet. Gr. Schaf & & 11 & 11 \\
\hline indet. Gr. Schwein & & 5,4 & 5,4 \\
\hline Total Unbestimmbare & 0 & 28,6 & 28,6 \\
\hline Gesamtergebnis & 4,4 & 403,4 & 407,8 \\
\hline
\end{tabular}

Tab. 16 Brugg-Kabelwerke 2007-2008 (Bru.007.3). Tierartentabelle (g), Strukturen Phase 3. 


\begin{tabular}{|c|c|c|c|c|c|c|c|c|c|c|c|c|c|c|c|c|}
\hline \multirow{3}{*}{ Bos taurus } & \multicolumn{16}{|l|}{ Phase 1} \\
\hline & \multicolumn{2}{|c|}{ Bachbett } & \multicolumn{2}{|c|}{$\begin{array}{l}\text { breiter Graben } \\
\text { westl. Tempel }\end{array}$} & \multicolumn{2}{|l|}{ Grube 8} & \multicolumn{2}{|c|}{ Pfostenreihe } & \multicolumn{2}{|c|}{$\begin{array}{l}\text { Schichten bei } \\
\text { cella }\end{array}$} & \multicolumn{2}{|c|}{$\begin{array}{l}\text { schmaler } \\
\text { Graben westl. } \\
\text { Tempel }\end{array}$} & \multicolumn{2}{|c|}{$\begin{array}{l}\text { Umfassungs- } \\
\text { graben }\end{array}$} & \multicolumn{2}{|c|}{ Phase 1 Ergebnis } \\
\hline & n & g & $\mathrm{n}$ & g & n & g & n & g & $\mathrm{n}$ & g & $\mathrm{n}$ & g & n & g & n & g \\
\hline Cranium & 1 & 166,1 & 1 & 1,1 & & & & & & & & & & & 2 & 167,2 \\
\hline \multicolumn{17}{|l|}{ Maxilla } \\
\hline loser Oberkieferzahn & 2 & 57,8 & & & & & & & & & & & & & 2 & 57,8 \\
\hline Mandibula & 9 & 436,4 & & & & & & & 2 & 17,1 & & & 2 & 9,9 & 13 & 463,4 \\
\hline Ioser Unterkieferzahn & 1 & 4,8 & & & & & & & 3 & 46,3 & & & & & 4 & 51,1 \\
\hline Unter-/Oberkieferzahn & 4 & 23,3 & & & & & & & 1 & 1,3 & 5 & 8,3 & & & 10 & 32,9 \\
\hline Total Kopf & 17 & 688,4 & 1 & 1,1 & 0 & 0 & 0 & 0 & 6 & 64,7 & 5 & 8,3 & 2 & 9,9 & 31 & 772,4 \\
\hline Atlas & 2 & 20,9 & & & & & & & & & 2 & 24,4 & & & 4 & 45,3 \\
\hline Epistropheus & 1 & 32,6 & & & & & & & & & & & & & 1 & 32,6 \\
\hline Vertebra cervicalis & & & & & & & & & 1 & 9,8 & & & & & 1 & 9,8 \\
\hline Vertebra thoracalis & 3 & 41,9 & 1 & 12,4 & & & & & 4 & 57,3 & & & 1 & 2,4 & 9 & 114 \\
\hline Vertebra lumbalis & 7 & 177,2 & 1 & 24,3 & & & & & 2 & 15 & & & & & 10 & 216,5 \\
\hline \multicolumn{17}{|l|}{ Sacrum } \\
\hline Vertebra indet. & 1 & 17,6 & & & & & & & & & 1 & 2,6 & & & 2 & 20,2 \\
\hline Costa & 12 & 70 & 4 & 48,7 & & & 3 & 10 & 43 & 302,2 & 9 & 119,5 & 6 & 36,8 & 77 & 587,2 \\
\hline \multicolumn{17}{|l|}{ Sternum } \\
\hline Total Rumpf & 26 & 360,2 & 6 & 85,4 & 0 & 0 & 3 & 10 & 50 & 384,3 & 12 & 146,5 & 7 & 39,2 & 104 & 1025,6 \\
\hline Scapula & 27 & 842,2 & 6 & 302,7 & & & & & 1 & 20,1 & 3 & 246,3 & 2 & 20,2 & 39 & 1431,5 \\
\hline Humerus & 20 & 738 & 10 & 491,3 & & & 1 & 17,4 & 3 & 53,2 & & & 2 & 51,4 & 36 & 1351,3 \\
\hline Pelvis & 4 & 90,6 & 2 & 169,3 & & & 6 & 116,2 & 2 & 34 & 2 & 41,9 & 2 & 31,9 & 18 & 483,9 \\
\hline Femur & 5 & 74,6 & 8 & 195 & & & & & 3 & 33,2 & 3 & 123,6 & 1 & 11,3 & 20 & 437,7 \\
\hline Patella & & & & & & & & & & & & & 1 & 10,7 & 1 & 10,7 \\
\hline Total Stylopodium & 56 & 1745,4 & 26 & 1158,3 & 0 & 0 & 7 & 133,6 & 9 & 140,5 & 8 & 411,8 & 8 & 125,5 & 114 & 3715,1 \\
\hline Radius & 3 & 105,9 & 7 & 169,8 & & & & & 2 & 15,9 & & & & & 12 & 291,6 \\
\hline Ulna & 1 & 4,7 & 1 & 42,2 & & & 1 & 2,6 & 3 & 11,3 & & & & & 6 & 60,8 \\
\hline Radius/UIna & 1 & 8,6 & 1 & 56,9 & & & & & & & & & & & 2 & 65,5 \\
\hline Tibia & 3 & 197,6 & 1 & 14,7 & & & & & 3 & 26,7 & 1 & 12,1 & & & 8 & 251,1 \\
\hline Total Zygopodium & 8 & 316,8 & 10 & 283,6 & 0 & 0 & 1 & 2,6 & 8 & 53,9 & 1 & 12,1 & 0 & 0 & 28 & 669 \\
\hline Carpalia & 1 & 9,2 & & & & & 1 & 18,4 & 1 & 6,4 & & & & & 3 & 34 \\
\hline Astragalus & & & 6 & 39,3 & & & & & & & & & & & 6 & 39,3 \\
\hline Calcaneus & 3 & 94,7 & 1 & 73,2 & & & & & 2 & 12,6 & & & & & 6 & 180,5 \\
\hline restl. Tarsalia & & & & & & & & & 1 & 9,2 & & & & & 1 & 9,2 \\
\hline $\mathrm{Mc} I \mathrm{II}+\mathrm{IV}$ & 7 & 467,7 & & & & & & & 4 & 167,4 & & & & & 11 & 635,1 \\
\hline Mt III+IV & 8 & 400,1 & 3 & 38,5 & 2 & 5,7 & & & 2 & 40 & 1 & 111,3 & 2 & 112,2 & 18 & 707,8 \\
\hline Hauptstrahl-Mp unbest. & 4 & 29,2 & & & & & & & & & & & & & 4 & 29,2 \\
\hline Phal. 1 ant. & & & & & & & & & 1 & 29,2 & & & & & 1 & 29,2 \\
\hline Phal. 1 ant./post. & 1 & 8,2 & & & & & & & 3 & 21,9 & & & 1 & 8,6 & 5 & 38,7 \\
\hline Phal. 1 post. & & & 1 & 19,6 & & & & & 2 & 37,4 & & & & & 3 & 57 \\
\hline Phal. 2 ant./post. & & & 1 & 11,6 & & & & & 2 & 39,6 & & & 2 & 35,7 & 5 & 86,9 \\
\hline Phal. 3 ant./post. & 1 & 4,7 & & & & & & & 2 & 27,6 & & & 1 & 11,3 & 4 & 43,6 \\
\hline Total Autopodium & 25 & 1013,8 & 12 & 182,2 & 2 & 5,7 & 1 & 18,4 & 20 & 391,3 & 1 & 111,3 & 6 & 167,8 & 67 & 1890,5 \\
\hline Röhrenknochen indet. & 5 & 43,9 & 1 & 8,9 & & & & & & & & & & & 6 & 52,8 \\
\hline Gesamtergebnis & 137 & 4168,5 & 56 & 1719,5 & 2 & 5,7 & 12 & 164,6 & 93 & 1034,7 & 27 & 690 & 23 & 342,4 & 350 & 8125,4 \\
\hline
\end{tabular}

Tab. 17 Brugg-Kabelwerke 2007-2008 (Bru.007.3). Skelettteilspektrum Bos taurus in den Strukturen 1-3. 


\begin{tabular}{|c|c|c|c|c|c|c|c|c|c|c|c|c|c|c|c|c|c|}
\hline \multicolumn{12}{|l|}{ Phase 2} & \multicolumn{4}{|l|}{ Phase 3} & \multirow{2}{*}{\multicolumn{2}{|c|}{ Gesamtergebnis }} \\
\hline \multicolumn{2}{|c|}{ Grube 23} & \multicolumn{2}{|l|}{ Grube 7} & \multicolumn{2}{|c|}{ Kapelle? } & \multicolumn{2}{|c|}{ Pfostenreihe } & \multicolumn{2}{|c|}{$\begin{array}{l}\text { Schichten bei } \\
\text { cella }\end{array}$} & \multicolumn{2}{|c|}{ Phase 2 Ergebnis } & \multicolumn{2}{|c|}{ Grube 22} & \multicolumn{2}{|c|}{ Phase 3 Ergebnis } & & \multirow[b]{2}{*}{ g } \\
\hline$n$ & $g$ & $\mathrm{n}$ & $g$ & $n$ & $g$ & $n$ & g & $n$ & $g$ & $n$ & $g$ & $n$ & g & $\mathrm{n}$ & g & $\mathrm{n}$ & \\
\hline & & & & & & & & & & & & & & & & 2 & 167,2 \\
\hline & & & & & & 1 & 27,9 & & & 1 & 27,9 & & & & & 1 & 27,9 \\
\hline & & & & & & & & 2 & 36,6 & 2 & 36,6 & & & & & 4 & 94,4 \\
\hline & & & & & & & & & & & & & & & & 13 & 463,4 \\
\hline & & 1 & 24,1 & & & & & & & 1 & 24,1 & & & & & 5 & 75,2 \\
\hline & & & & 1 & 0,4 & & & & & 1 & 0,4 & & & & & 11 & 33,3 \\
\hline \multirow[t]{5}{*}{0} & 0 & 1 & 24,1 & 1 & 0,4 & 1 & 27,9 & 2 & 36,6 & 5 & 89 & 0 & 0 & 0 & 0 & 36 & 861,4 \\
\hline & & & & & & 1 & 34,2 & & & 1 & 34,2 & & & & & 5 & 79,5 \\
\hline & & & & & & & & & & & & & & & & 1 & 32,6 \\
\hline & & 1 & 5,1 & & & & & 1 & 12,5 & 2 & 17,6 & & & & & 3 & 27,4 \\
\hline & & & & & & 6 & 70,5 & 2 & 32,2 & 8 & 102,7 & & & & & 17 & 216,7 \\
\hline \multirow[t]{3}{*}{1} & 7,1 & & & & & & & 1 & 8,1 & 2 & 15,2 & & & & & 12 & 231,7 \\
\hline & & & & & & 1 & 42 & & & 1 & 42 & & & & & 1 & 42 \\
\hline & & & & & & & & 1 & 1,9 & 1 & 1,9 & & & & & 3 & 22,1 \\
\hline \multirow[t]{2}{*}{3} & 24,9 & 1 & 62,2 & 2 & 11,7 & 21 & 330,7 & 8 & 53,4 & 35 & 482,9 & 7 & 44,7 & 7 & 44,7 & 119 & 1114,8 \\
\hline & & & & & & & & & & & & 1 & 4,5 & 1 & 4,5 & 1 & 4,5 \\
\hline \multirow[t]{6}{*}{4} & 32 & 2 & 67,3 & 2 & 11,7 & 29 & 477,4 & 13 & 108,1 & 50 & 696,5 & 8 & 49,2 & 8 & 49,2 & 162 & 1771,3 \\
\hline & & & & 1 & 36,5 & 1 & 28,3 & & & 2 & 64,8 & & & & & 41 & 1496,3 \\
\hline & & 2 & 37,8 & & & 6 & 177,3 & 5 & 87 & 13 & 302,1 & & & & & 49 & 1653,4 \\
\hline & & & & 1 & 24,2 & 4 & 171,7 & & & 5 & 195,9 & 1 & 15,4 & 1 & 15,4 & 24 & 695,2 \\
\hline & & & & & & 2 & 60,1 & & & 2 & 60,1 & & & & & 22 & 497,8 \\
\hline & & & & & & 1 & 22,6 & & & 1 & 22,6 & & & & & 2 & 33,3 \\
\hline 0 & 0 & 2 & 37,8 & 2 & 60,7 & 14 & 460 & 5 & 87 & 23 & 645,5 & 1 & 15,4 & 1 & 15,4 & 138 & 4376 \\
\hline & & & & & & 1 & 6,9 & & & 1 & 6,9 & & & & & 13 & 298,5 \\
\hline & & & & & & 1 & 10,6 & & & 1 & 10,6 & & & & & 7 & 71,4 \\
\hline & & & & & & & & & & & & & & & & 2 & 65,5 \\
\hline & & & & & & 2 & 61,8 & & & 2 & 61,8 & 1 & 13,8 & 1 & 13,8 & 11 & 326,7 \\
\hline 0 & 0 & 0 & 0 & 0 & 0 & 4 & 79,3 & 0 & 0 & 4 & 79,3 & 1 & 13,8 & 1 & 13,8 & 33 & 762,1 \\
\hline & & & & & & 2 & 24,1 & 1 & 10,3 & 3 & 34,4 & 1 & 4,8 & 1 & 4,8 & 7 & 73,2 \\
\hline & & & & & & & & & & & & 1 & 27,1 & 1 & 27,1 & 7 & 66,4 \\
\hline & & & & & & 1 & 20,6 & & & 1 & 20,6 & & & & & 7 & 201,1 \\
\hline & & & & & & 1 & 11,3 & & & 1 & 11,3 & & & & & 2 & 20,5 \\
\hline & & & & & & 3 & 112,4 & 1 & 84,9 & 4 & 197,3 & & & & & 15 & 832,4 \\
\hline & & 1 & 6,9 & & & 4 & 123,4 & 2 & 23,3 & 7 & 153,6 & & & & & 25 & 861,4 \\
\hline & & & & & & 1 & 2,3 & & & 1 & 2,3 & & & & & 5 & 31,5 \\
\hline & & & & & & & & & & & & 1 & 26,4 & 1 & 26,4 & 2 & 55,6 \\
\hline & & & & & & 2 & 27,2 & 1 & 16,1 & 3 & 43,3 & 1 & 4,2 & 1 & 4,2 & 9 & 86,2 \\
\hline & & & & & & & & & & & & & & & & 3 & 57 \\
\hline & & & & & & 2 & 24,3 & & & 2 & 24,3 & & & & & 7 & 111,2 \\
\hline & & & & & & 1 & 17,9 & 1 & 20,6 & 2 & 38,5 & & & & & 6 & 82,1 \\
\hline 0 & 0 & 1 & 6,9 & 0 & 0 & 17 & 363,5 & 6 & 155,2 & 24 & 525,6 & 4 & 62,5 & 4 & 62,5 & 95 & 2478,6 \\
\hline & & & & & & & & & & & & & & & & 6 & 52,8 \\
\hline 4 & 32 & 6 & 136,1 & 5 & 72,8 & 65 & 1408,1 & 26 & 386,9 & 106 & 2035,9 & 14 & 140,9 & 14 & 140,9 & 470 & 10302,2 \\
\hline
\end{tabular}




\begin{tabular}{|c|c|c|c|c|c|c|c|c|c|c|c|c|c|c|c|c|c|c|}
\hline \multirow{3}{*}{ Sus domesticus } & \multicolumn{18}{|l|}{ Phase 1} \\
\hline & \multicolumn{2}{|c|}{ Bachbett } & \multicolumn{2}{|c|}{$\begin{array}{l}\text { breiter Graben } \\
\text { westlich T. }\end{array}$} & \multicolumn{2}{|l|}{ Grube 1} & \multicolumn{2}{|c|}{ Grube 15} & \multicolumn{2}{|c|}{ Grube 20} & \multicolumn{2}{|l|}{ Grube 8} & \multicolumn{2}{|c|}{ Pfostenreihe } & \multicolumn{2}{|c|}{$\begin{array}{l}\text { Schichten bei } \\
\text { cella }\end{array}$} & \multicolumn{2}{|c|}{$\begin{array}{l}\text { schmaler Graben } \\
\text { westlich T. }\end{array}$} \\
\hline & $\mathrm{n}$ & g & n & $g$ & $\mathrm{n}$ & $g$ & $\mathrm{n}$ & $g$ & $\mathrm{n}$ & $g$ & $\mathrm{n}$ & $\mathrm{g}$ & $n$ & $g$ & $\mathrm{n}$ & g & $\mathrm{n}$ & g \\
\hline Cranium & 2 & 7,2 & & & & & & & & & & & 2 & 8,1 & 7 & 21,3 & 1 & 2,5 \\
\hline Maxilla & & & & & & & 1 & 8,4 & & & & & & & & & & \\
\hline loser Oberkieferzahn & 4 & 5,4 & & & & & & & & & & & 1 & 0,2 & 11 & 24,7 & & \\
\hline Mandibula & 5 & 204,8 & 1 & 3,4 & & & 3 & 16,4 & 1 & 2 & & & & & 12 & 51,7 & 4 & 6,3 \\
\hline loser Unterkieferzahn & 6 & 21,6 & & & & & 2 & 2,3 & & & & & & & 12 & 24,8 & 2 & 6,7 \\
\hline Unter-/0berkieferfragm. & 1 & 0,1 & & & & & 1 & 0,2 & & & & & & & & & & \\
\hline Unter-/Oberkieferzahn & 1 & 0,2 & & & 1 & 0,1 & 1 & 0,2 & & & & & & & & & & \\
\hline Total Kopf & 19 & 239,3 & 1 & 3,4 & 1 & 0,1 & 8 & 27,5 & 1 & 2 & 0 & 0 & 3 & 8,3 & 42 & 122,5 & 7 & 15,5 \\
\hline Atlas & 1 & 1,1 & & & & & & & 1 & 3,9 & & & & & & & & \\
\hline Epistropheus & & & & & & & & & & & & & & & 1 & 3,5 & & \\
\hline Vertebra thoracalis & 1 & 2,3 & & & & & & & & & & & & & 2 & 6,3 & & \\
\hline Vertebra lumbalis & & & & & & & & & & & & & & & 3 & 7,2 & & \\
\hline Vertebra caudalis & 1 & 0,5 & & & & & & & & & & & & & & & & \\
\hline Costa & 6 & 9,2 & & & 1 & 1,1 & 4 & 5,3 & & & . & & 3 & 5,1 & 21 & 42,9 & 1 & 1,1 \\
\hline Total Rumpf & 9 & 13,1 & 0 & 0 & 1 & 1,1 & 4 & 5,3 & 1 & 3,9 & 0 & 0 & 3 & 5,1 & 27 & 59,9 & 1 & 1,1 \\
\hline Scapula & 6 & 16,8 & 1 & 3,9 & & & & & & & & & 1 & 8,7 & 7 & 34,8 & & \\
\hline Humerus & 3 & 37,7 & & & & & 2 & 11,3 & & & 2 & 8,9 & & & 18 & 87,1 & 3 & 8,4 \\
\hline Pelvis & 3 & 25,7 & & & 1 & 4,5 & & & 1 & 1,2 & & & 2 & 22,2 & 6 & 45,3 & 1 & 9,2 \\
\hline Femur & 1 & 5,4 & 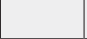 & & 4 & 20,6 & & & & & 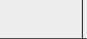 & & & & 14 & 83,4 & & \\
\hline Total Stylopodium & 13 & 85,6 & 1 & 3,9 & 5 & 25,1 & 2 & 11,3 & 1 & 1,2 & 2 & 8,9 & 3 & 30,9 & 45 & 250,6 & 4 & 17,6 \\
\hline Radius & & & & & & & 1 & 0,5 & & & & & 1 & 2 & 7 & 22,9 & & \\
\hline Ulna & & & & & & & & & & & & & & & 4 & 45,9 & 1 & 7,3 \\
\hline Tibia & 7 & 46,4 & 1 & 20,3 & 6 & 48,2 & 1 & 1,5 & & & & & 1 & 2,7 & 12 & 67,5 & 1 & 7,6 \\
\hline Fibula & & & & & & & & & 2 & 2,5 & & & 1 & 1,2 & 1 & 0,4 & & \\
\hline Total Zygopodium & 7 & 46,4 & 1 & 20,3 & 6 & 48,2 & 2 & 2 & 2 & 2,5 & 0 & 0 & 3 & 5,9 & 24 & 136,7 & 2 & 14,9 \\
\hline Astragalus & & & 1 & 8 & 1 & 6,1 & & & & & & & & & & & & \\
\hline Calcaneus & 1 & 10,5 & & & & & 1 & 1,1 & & & & & & & 1 & 10 & & \\
\hline restl. Tarsalia & & & & & & & & & & & & & & & 1 & 8,8 & & \\
\hline Mc IV & & & & & & & & & & & & & & & 2 & 5,4 & & \\
\hline \multicolumn{19}{|l|}{ Mc V } \\
\hline \multicolumn{19}{|l|}{ Mt IV } \\
\hline Nebenstrahl-Mp unbest. & 1 & 0,5 & & & & & & & & & & & & & 1 & 0,9 & & \\
\hline Hauptstrahl-Mp unbest. & & & & & & & 1 & 0,3 & & & & & & & 1 & 2,9 & 1 & 0,2 \\
\hline Phal. 1 ant./post. & 1 & 1 & & & & & & & & & & & & & 4 & 8,3 & 1 & 2,7 \\
\hline Phal. 2 ant./post. & & & & & & & & & & & & & & & 2 & 2,3 & & \\
\hline Total Autopodium & 3 & 12 & 1 & 8 & 1 & 6,1 & 2 & 1,4 & 0 & 0 & 0 & 0 & 0 & 0 & 12 & 38,6 & 2 & 2,9 \\
\hline Plattenknochen & 1 & 2,4 & & & & & & & & & & & & & & & & \\
\hline Gesamtergebnis & 52 & 398,8 & 4 & 35,6 & 14 & 80,6 & 18 & 47,5 & 5 & 9,6 & 2 & 8,9 & 12 & 50,2 & 150 & 608,3 & 16 & 52 \\
\hline
\end{tabular}

Tab. 18 Brugg-Kabelwerke 2007-2008 (Bru.007.3). Skelettteilspektrum Sus domesticus in den Strukturen 1-3. 


\begin{tabular}{|c|c|c|c|c|c|c|c|c|c|c|c|c|c|c|c|c|c|c|c|c|c|c|c|}
\hline \multirow{2}{*}{\multicolumn{2}{|c|}{$\begin{array}{l}\text { Umfassungs- } \\
\text { graben }\end{array}$}} & \multirow{2}{*}{\multicolumn{2}{|c|}{$\begin{array}{l}\text { Phase } 1 \\
\text { Ergebnis }\end{array}$}} & \multicolumn{12}{|l|}{ Phase 2} & \multicolumn{6}{|l|}{ Phase 3} & \multirow{2}{*}{\multicolumn{2}{|c|}{ Gesamtergebnis }} \\
\hline & & & & \multicolumn{2}{|c|}{ Grube 23} & \multicolumn{2}{|c|}{ Grube 7} & \multicolumn{2}{|c|}{ Kapelle? } & \multicolumn{2}{|c|}{ Pfostenreihe } & \multicolumn{2}{|c|}{$\begin{array}{l}\text { Schichten bei } \\
\text { cella }\end{array}$} & \multicolumn{2}{|c|}{\begin{tabular}{|l} 
Phase 2 \\
Ergebnis
\end{tabular}} & \multicolumn{2}{|c|}{ Grube 16} & \multicolumn{2}{|c|}{ Grube 22} & \multicolumn{2}{|c|}{\begin{tabular}{|l} 
Phase 3 \\
Ergebnis
\end{tabular}} & & \\
\hline$n$ & g & $\mathrm{n}$ & g & $\mathrm{n}$ & g & $\mathrm{n}$ & g & $\mathrm{n}$ & g & $\mathrm{n}$ & g & $\mathrm{n}$ & g & $\mathrm{n}$ & g & $\mathrm{n}$ & $g$ & $n$ & g & $\mathrm{n}$ & g & $n$ & g \\
\hline & & 12 & 39,1 & & & & & 1 & 1,3 & 20 & 70,1 & & & 21 & 71,4 & & & 2 & 5,7 & 2 & 5,7 & 35 & 116,2 \\
\hline 1 & 5,9 & 2 & 14,3 & 1 & 0,8 & & & & & 3 & 39,4 & & & 4 & 40,2 & & & & & & & 6 & 54,5 \\
\hline 3 & 4,3 & 19 & 34,6 & & & & & 1 & 0,5 & 2 & 10,5 & 2 & 5,3 & 5 & 16,3 & & & & & & & 24 & 50,9 \\
\hline 3 & 7,9 & 29 & 292,5 & 1 & 0,9 & & & 1 & 0,5 & 10 & 103,5 & 8 & 74,3 & 20 & 179,2 & & & 4 & 25,9 & 4 & 25,9 & 53 & 497,6 \\
\hline \multirow[t]{3}{*}{5} & 7,8 & 27 & 63,2 & 1 & 6,8 & & & & & 2 & 3,5 & 4 & 17,4 & 7 & 27,7 & & & 1 & 0,6 & 1 & 0,6 & 35 & 91,5 \\
\hline & & 2 & 0,3 & & & & & & & 2 & 1,1 & 2 & 1,8 & 4 & 2,9 & & & & & & & 6 & 3,2 \\
\hline & & 3 & 0,5 & & & & & & & 1 & 1,7 & 1 & 0,3 & 2 & 2 & & & & & & & 5 & 2,5 \\
\hline \multirow[t]{3}{*}{12} & 25,9 & 94 & 444,5 & 3 & 8,5 & 0 & 0 & 3 & 2,3 & 40 & 229,8 & 17 & 99,1 & 63 & 339,7 & 0 & 0 & 7 & 32,2 & 7 & 32,2 & 164 & 816,4 \\
\hline & & 2 & 5 & & & & & & & & & & & & & & & & & & & 2 & 5 \\
\hline & & 1 & 3,5 & & & & & & & & & & & & & & & & & & & 1 & 3,5 \\
\hline \multirow[t]{3}{*}{1} & 1,7 & 4 & 10,3 & & & & & & & & & & & & & & & & & & & 4 & 10,3 \\
\hline & & 3 & 7,2 & & & & & & & 1 & 4,4 & & & 1 & 4,4 & & & & & & & 4 & 11,6 \\
\hline & & 1 & 0,5 & & & & & & & & & & & & & & & & & & & 1 & 0,5 \\
\hline 2 & 4 & 38 & 68,7 & 2 & 4,3 & 2 & 4,5 & & & 9 & 20,8 & 2 & 5,8 & 15 & 35,4 & & & 9 & 10,2 & 9 & 10,2 & 62 & 114,3 \\
\hline 3 & 5,7 & 49 & 95,2 & 2 & 4,3 & 2 & 4,5 & 0 & 0 & 10 & 25,2 & 2 & 5,8 & 16 & 39,8 & 0 & 0 & 9 & 10,2 & 9 & 10,2 & 74 & 145,2 \\
\hline 4 & 13,3 & 19 & 77,5 & & & & & 1 & 1,5 & 18 & 61,2 & 4 & 27,2 & 23 & 89,9 & & & 2 & 16,6 & 2 & 16,6 & 44 & 184 \\
\hline 1 & 3,4 & 29 & 156,8 & 1 & 5,4 & 1 & 1,4 & 1 & 1 & 3 & 10 & 5 & 34,8 & 11 & 52,6 & & & 4 & 24,3 & 4 & 24,3 & 44 & 233,7 \\
\hline 1 & 8,5 & 15 & 116,6 & & & & & & & 4 & 46,3 & & & 4 & 46,3 & & & 1 & 9,5 & 1 & 9,5 & 20 & 172,4 \\
\hline & & 19 & 109,4 & & & & & 1 & 2,5 & 7 & 55,2 & 3 & 13,1 & 11 & 70,8 & & & 4 & 33,2 & 4 & 33,2 & 34 & 213,4 \\
\hline 6 & 25,2 & 82 & 460,3 & 1 & 5,4 & 1 & 1,4 & 3 & 5 & 32 & 172,7 & 12 & 75,1 & 49 & 259,6 & 0 & 0 & 11 & 83,6 & 11 & 83,6 & 142 & 803,5 \\
\hline 2 & 12,6 & 11 & 38 & 1 & 0,7 & & & & & 1 & 0,9 & 2 & 9,2 & 4 & 10,8 & & & 4 & 10,6 & 4 & 10,6 & 19 & 59,4 \\
\hline 3 & 21,1 & 8 & 74,3 & & & & & & & 1 & 1,4 & 1 & 4,8 & 2 & 6,2 & 1 & 2,7 & 2 & 11,6 & 3 & 14,3 & 13 & 94,8 \\
\hline 4 & 34,6 & 33 & 228,8 & 1 & 9,8 & & & 3 & 12,8 & 6 & 39,6 & 3 & 9,8 & 13 & 72 & & & 4 & 16,6 & 4 & 16,6 & 50 & 317,4 \\
\hline & & 4 & 4,1 & 1 & 0,4 & & & & & & & & & 1 & 0,4 & & & 6 & 5,8 & 6 & 5,8 & 11 & 10,3 \\
\hline 9 & 68,3 & 56 & 345,2 & 3 & 10,9 & 0 & 0 & 3 & 12,8 & 8 & 41,9 & 6 & 23,8 & 20 & 89,4 & 1 & 2,7 & 16 & 44,6 & 17 & 47,3 & 93 & 481,9 \\
\hline & & 2 & 14,1 & & & & & & & & & & & & & & & & & & & 2 & 14,1 \\
\hline & & 3 & 21,6 & & & & & & & & & & & & & & & & & & & 3 & 21,6 \\
\hline & & 1 & 8,8 & & & & & & & & & & & & & & & & & & & 1 & 8,8 \\
\hline & & 2 & 5,4 & & & & & & & & & & & & & & & & & & & 2 & 5,4 \\
\hline & & & & & & & & & & & & & & & & & & 1 & 2,3 & 1 & 2,3 & 1 & 2,3 \\
\hline & & & & & & & & 1 & 1,2 & & & 1 & 2,3 & 2 & 3,5 & & & & & & & 2 & 3,5 \\
\hline & & 2 & 1,4 & & & & & & & & & 2 & 1,8 & 2 & 1,8 & & & 1 & 1,2 & 1 & 1,2 & 5 & 4,4 \\
\hline & & 3 & 3,4 & & & & & & & 1 & 4,6 & 1 & 1,1 & 2 & 5,7 & & & 2 & 5 & 2 & 5 & 7 & 14,1 \\
\hline & & 6 & 12 & & & & & 1 & 1,5 & & & & & 1 & 1,5 & & & & & & & 7 & 13,5 \\
\hline & & 2 & 2,3 & & & & & & & 2 & 2,9 & & & 2 & 2,9 & & & & & & & 4 & 5,2 \\
\hline 0 & 0 & 21 & 69 & 0 & 0 & 0 & 0 & 2 & 2,7 & 3 & 7,5 & 4 & 5,2 & 9 & 15,4 & 0 & 0 & 4 & 8,5 & 4 & 8,5 & 34 & 92,9 \\
\hline & & 1 & 2,4 & & & & & & & & & & & & & & & & & & & 1 & 2,4 \\
\hline 30 & 125,1 & 303 & 1416,6 & 9 & 29,1 & 3 & 5,9 & 11 & 22,8 & 93 & 477,1 & 41 & 209 & 157 & 743,9 & 1 & 2,7 & 47 & 179,1 & 48 & 181,8 & 508 & 2342,3 \\
\hline
\end{tabular}




\begin{tabular}{|c|c|c|c|c|c|c|c|c|c|c|c|c|c|c|c|c|}
\hline \multirow{3}{*}{ Ovis/Capra } & \multicolumn{16}{|l|}{ Phase 1} \\
\hline & \multicolumn{2}{|l|}{ Bachbett } & \multicolumn{2}{|l|}{ Grube 1} & \multicolumn{2}{|l|}{ Grube 15} & \multicolumn{2}{|c|}{ Grube 20} & \multicolumn{2}{|c|}{ Pfostenreihe } & \multicolumn{2}{|c|}{$\begin{array}{l}\text { Schichten bei } \\
\text { cella }\end{array}$} & \multicolumn{2}{|c|}{$\begin{array}{l}\text { schmaler Graben } \\
\text { westl. Tempel }\end{array}$} & \multicolumn{2}{|c|}{$\begin{array}{l}\text { Umfassungsgra- } \\
\text { ben }\end{array}$} \\
\hline & $\mathrm{n}$ & $g$ & $\mathrm{n}$ & $g$ & $\mathrm{n}$ & $g$ & $\mathrm{n}$ & $g$ & $\mathrm{n}$ & g & $\mathrm{n}$ & g & $\mathrm{n}$ & g & $\mathrm{n}$ & g \\
\hline Cranium & & & & & & & & & & & 1 & 0,5 & & & & \\
\hline Maxilla & & & & & & & & & & & 1 & 0,9 & & & 1 & 9,6 \\
\hline loser Oberkieferzahn & 5 & 25,4 & & & & & & & & & 5 & 10,7 & & & 5 & 22,4 \\
\hline Mandibula & 5 & 18,3 & & & & & & & & & 15 & 76,1 & 1 & 36,6 & 5 & 8,5 \\
\hline loser Unterkieferzahn & 4 & 9,4 & & & & & 1 & 0,2 & & & 14 & 35,3 & & & 6 & 25,6 \\
\hline Unter- oder Oberkieferzahn & 1 & 0,3 & & & & & & & & & 8 & 2,6 & & & & \\
\hline Total Kopf & 15 & 53,4 & 0 & 0 & 0 & 0 & 1 & 0,2 & 0 & 0 & 44 & 126,1 & 1 & 36,6 & 17 & 66,1 \\
\hline Atlas & & & & & & & & & & & 2 & 9,6 & & & & \\
\hline Vertebra cervicalis & & & & & & & & & & & 1 & 1,1 & & & & \\
\hline Vertebra thoracalis & & & & & & & & & & & 1 & 2 & & & & \\
\hline Vertebra lumbalis & & & & & & & & & & & 3 & 12,3 & & & & \\
\hline Vertebra indet. & & & & & & & & & & & & & 1 & 1,3 & & \\
\hline Costa & & & & & 1 & 1,1 & & & & & 10 & 10,4 & & & & \\
\hline Total Rumpf & 0 & 0 & 0 & 0 & 1 & 1,1 & 0 & 0 & 0 & 0 & 17 & 35,4 & 1 & 1,3 & 0 & 0 \\
\hline Scapula & & & & & & & & & & & 4 & 6,5 & 1 & 4,2 & 1 & 1,1 \\
\hline Humerus & 3 & 10,6 & 1 & 1,1 & & & & & 2 & 9,9 & 18 & 73,1 & 2 & 17 & 4 & 8,4 \\
\hline Pelvis & & & & & & & & & & & 1 & 6,2 & & & & \\
\hline Femur & 2 & 2,9 & & & & & & & 1 & 1,9 & 18 & 34,3 & & & 1 & 6,8 \\
\hline Total Stylopodium & 5 & 13,5 & 1 & 1,1 & 0 & 0 & 0 & 0 & 3 & 11,8 & 41 & 120,1 & 3 & 21,2 & 6 & 16,3 \\
\hline Radius & 1 & 4,1 & & & & & & & 1 & 3,9 & 16 & 38,6 & & & 3 & 9,6 \\
\hline \multicolumn{17}{|l|}{ Ulna } \\
\hline Radius/Ulna & & & & & & & & & & & 1 & 3,9 & & & & \\
\hline Tibia & & & & & 1 & 3,4 & & & & & 18 & 115,6 & 1 & 12,2 & 6 & 22,5 \\
\hline Total Zygopodium & 1 & 4,1 & 0 & 0 & 1 & 3,4 & 0 & 0 & 1 & 3,9 & 35 & 158,1 & 1 & 12,2 & 9 & 32,1 \\
\hline Astragalus & & & & & & & & & & & 1 & 3,3 & & & & \\
\hline Calcaneus & & & & & & & & & & & 3 & 10,6 & & & & \\
\hline Centrotarsale & & & & & & & & & & & & & & & 1 & 1,8 \\
\hline Mc III+IV & 1 & 4,7 & & & & & & & 1 & 5,6 & 3 & 6,1 & & & & \\
\hline Mt III+IV & 2 & 2,7 & & & & & & & & & 2 & 2,6 & & & & \\
\hline Hauptstrahl-Mp unbestimmbar & & & & & & & & & & & 2 & 2,1 & & & & \\
\hline Phal. 2 ant./post. & & & & & & & & & & & 1 & 1,1 & & & & \\
\hline Total Autopodium & 3 & 7,4 & 0 & 0 & 0 & 0 & 0 & 0 & 1 & 5,6 & 12 & 25,8 & 0 & 0 & 1 & 1,8 \\
\hline Gesamtergebnis & 24 & 78,4 & 1 & 1,1 & 2 & 4,5 & 1 & 0,2 & 5 & 21,3 & 149 & 465,5 & 6 & 71,3 & 33 & 116,3 \\
\hline
\end{tabular}

Tab. 19 Brugg-Kabelwerke 2007-2008 (Bru.007.3). Skelettteilspektrum 0vis/Capra in den Strukturen 1-3. 


\begin{tabular}{|c|c|c|c|c|c|c|c|c|c|c|c|c|c|c|c|c|c|c|c|}
\hline & & Phase 2 & & & & & & & & & & & & Phase 3 & & & & Gesamte & ebnis \\
\hline Phase $1 \mathrm{E}$ & gebnis & Grube 23 & & Grube 7 & & Kapelle? & & Pfostenr & & Schichter & & Phase $2 \mathrm{E}$ & gebnis & Grube 22 & & Phase 3 & ebnis & & \\
\hline$n$ & g & $\mathrm{n}$ & g & $\mathrm{n}$ & $g$ & $\mathrm{n}$ & $g$ & $n$ & g & $\mathrm{n}$ & g & $n$ & g & $\mathrm{n}$ & $g$ & $\mathrm{n}$ & $g$ & $\mathrm{n}$ & g \\
\hline 1 & 0,5 & & & & & & & 2 & 3,8 & & & 2 & 3,8 & & & & & 3 & 4,3 \\
\hline 2 & 10,5 & & & & & & & 1 & 1,4 & & & 1 & 1,4 & & & & & 3 & 11,9 \\
\hline 15 & 58,5 & & & 2 & 10,9 & & & 4 & 16,4 & & & 6 & 27,3 & & & & & 21 & 85,8 \\
\hline 26 & 139,5 & 1 & 10,1 & & & 2 & 1,6 & 16 & 68,7 & & & 19 & 80,4 & & & & & 45 & 219,9 \\
\hline 25 & 70,5 & 2 & 7,9 & 1 & 3,1 & & & 6 & 11,4 & 1 & 0,6 & 10 & 23 & 1 & 2,1 & 1 & 2,1 & 36 & 95,6 \\
\hline 9 & 2,9 & & & 1 & 1,2 & 1 & 0,5 & 3 & 2,5 & & & 5 & 4,2 & & & & & 14 & 7,1 \\
\hline 78 & 282,4 & 3 & 18 & 4 & 15,2 & 3 & 2,1 & 32 & 104,2 & 1 & 0,6 & 43 & 140,1 & 1 & 2,1 & 1 & 2,1 & 122 & 424,6 \\
\hline 2 & 9,6 & & & & & & & 1 & 5,5 & & & 1 & 5,5 & & & & & 3 & 15,1 \\
\hline 1 & 1,1 & & & & & & & 1 & 5,1 & & & 1 & 5,1 & & & & & 2 & 6,2 \\
\hline 1 & 2 & & & & & & & & & & & & & 1 & 0,5 & 1 & 0,5 & 2 & 2,5 \\
\hline 3 & 12,3 & & & & & & & & & & & & & 1 & 2,3 & 1 & 2,3 & 4 & 14,6 \\
\hline 1 & 1,3 & & & & & & & & & & & & & & & & & 1 & 1,3 \\
\hline 11 & 11,5 & & & & & & & 4 & 1,6 & & & 4 & 1,6 & 7 & 6,9 & 7 & 6,9 & 22 & 20 \\
\hline 19 & 37,8 & 0 & 0 & 0 & 0 & 0 & 0 & 6 & 12,2 & 0 & 0 & 6 & 12,2 & 9 & 9,7 & 9 & 9,7 & 34 & 59,7 \\
\hline 6 & 11,8 & & & & & & & 2 & 8,6 & & & 2 & 8,6 & & & & & 8 & 20,4 \\
\hline 30 & 120,1 & & & & & 5 & 15,2 & 6 & 24,8 & & & 11 & 40 & 2 & 7,9 & 2 & 7,9 & 43 & 168 \\
\hline 1 & 6,2 & & & & & & & & & & & & & & & & & 1 & 6,2 \\
\hline 22 & 45,9 & 2 & 8 & & & 1 & 2,2 & 9 & 18 & 4 & 15,5 & 16 & 43,7 & 5 & 11,5 & 5 & 11,5 & 43 & 101,1 \\
\hline 59 & 184 & 2 & 8 & 0 & 0 & 6 & 17,4 & 17 & 51,4 & 4 & 15,5 & 29 & 92,3 & 7 & 19,4 & 7 & 19,4 & 95 & 295,7 \\
\hline 21 & 56,2 & & & & & 2 & 3,7 & 6 & 14,4 & 5 & 28,3 & 13 & 46,4 & 2 & 1,5 & 2 & 1,5 & 36 & 104,1 \\
\hline & & & & & & & & 1 & 0,9 & & & 1 & 0,9 & & & & & 1 & 0,9 \\
\hline 1 & 3,9 & & & & & & & & & & & & & & & & & 1 & 3,9 \\
\hline 26 & 153,7 & 1 & 6,9 & 1 & 4,1 & 3 & 4 & 8 & 51,6 & 4 & 16,1 & 17 & 82,7 & 5 & 18,4 & 5 & 18,4 & 48 & 254,8 \\
\hline 48 & 213,8 & 1 & 6,9 & 1 & 4,1 & 5 & 7,7 & 15 & 66,9 & 9 & 44,4 & 31 & 130 & 7 & 19,9 & 7 & 19,9 & 86 & 363,7 \\
\hline 1 & 3,3 & & & & & & & 1 & 3,6 & & & 1 & 3,6 & & & & & 2 & 6,9 \\
\hline 3 & 10,6 & & & & & & & & & & & & & & & & & 3 & 10,6 \\
\hline 1 & 1,8 & & & & & & & & & & & & & & & & & 1 & 1,8 \\
\hline 5 & 16,4 & & & & & & & & & 2 & 2,5 & 2 & 2,5 & & & & & 7 & 18,9 \\
\hline 4 & 5,3 & & & & & & & 1 & 1,7 & & & 1 & 1,7 & & & & & 5 & 7 \\
\hline 2 & 2,1 & & & & & & & & & & & & & & & & & 2 & 2,1 \\
\hline 1 & 1,1 & & & & & & & & & & & & & & & & & 1 & 1,1 \\
\hline 17 & 40,6 & 0 & 0 & 0 & 0 & 0 & 0 & 2 & 5,3 & 2 & 2,5 & 4 & 7,8 & 0 & 0 & 0 & 0 & 21 & 48,4 \\
\hline 221 & 758,6 & 6 & 32,9 & 5 & 19,3 & 14 & 27,2 & 72 & 240 & 16 & 63 & 113 & 382,4 & 24 & 51,1 & 24 & 51,1 & 358 & 1192,1 \\
\hline
\end{tabular}




\begin{tabular}{|c|c|c|c|c|c|c|c|c|c|c|}
\hline \multirow{3}{*}{ Equiden } & \multicolumn{10}{|l|}{ Phase 1} \\
\hline & \multicolumn{2}{|l|}{ Bachbett } & \multicolumn{2}{|c|}{$\begin{array}{l}\text { breiter Graben } \\
\text { westl. Tempel }\end{array}$} & \multicolumn{2}{|c|}{ Schichten bei Cella } & \multicolumn{2}{|c|}{$\begin{array}{l}\text { schmaler Graben } \\
\text { westl. Tempel }\end{array}$} & \multicolumn{2}{|c|}{ Phase 1 Ergebnis } \\
\hline & $\mathrm{n}$ & g & $\mathrm{n}$ & g & $\mathrm{n}$ & g & $\mathrm{n}$ & g & $\mathrm{n}$ & g \\
\hline Cranium & 1 & 8,8 & 1 & 13,6 & & & & & 2 & 22,4 \\
\hline loser Oberkieferzahn & & & 3 & 139,9 & 1 & 39,4 & & & 4 & 179,3 \\
\hline Mandibula & 5 & 196 & 3 & 490,1 & & & 2 & 471,5 & 10 & 1157,6 \\
\hline loser Unterkieferzahn & 4 & 133 & 1 & 36,5 & & & & & 5 & 169,5 \\
\hline Unter- oder Oberkieferzahn & 5 & 26,2 & & & & & & & 5 & 26,2 \\
\hline Total Schädel & 15 & 364 & 8 & 680,1 & 1 & 39,4 & 2 & 471,5 & 26 & 1555 \\
\hline Vertebra cervicalis & 5 & 161,1 & & & & & 3 & 53,6 & 8 & 214,7 \\
\hline Vertebra thoracalis & & & & & & & 1 & 10,6 & 1 & 10,6 \\
\hline Vertebra indet. & & & & & & & 1 & 4,7 & 1 & 4,7 \\
\hline Costa & 5 & 32,1 & & & & & & & 5 & 32,1 \\
\hline Total Rumpf & 10 & 193,2 & 0 & 0 & 0 & 0 & 5 & 68,9 & 15 & 262,1 \\
\hline Scapula & 1 & 144,1 & & & & & & & 1 & 144,1 \\
\hline Humerus & 12 & 774,4 & 1 & 200,7 & & & 3 & 57,9 & 16 & 1033 \\
\hline Pelvis & 5 & 377,3 & 1 & 117,5 & & & & & 6 & 494,8 \\
\hline Femur & 9 & 1209,2 & 4 & 442,1 & & & & & 13 & 1651,3 \\
\hline Total Stylopodium & 27 & 2505 & 6 & 760,3 & 0 & 0 & 3 & 57,9 & 36 & 3323,2 \\
\hline Ulna & 4 & 75 & & & & & & & 4 & 75 \\
\hline Radius & 4 & 421,1 & & & & & 3 & 85,7 & 7 & 506,8 \\
\hline Radius/UIna & 1 & 278,2 & 1 & 400,8 & & & 1 & 257,2 & 3 & 936,2 \\
\hline Tibia & 5 & 146,4 & & & & & & & 5 & 146,4 \\
\hline Total Zygopodium & 14 & 920,7 & 1 & 400,8 & 0 & 0 & 4 & 342,9 & 19 & 1664,4 \\
\hline Carpalia & 1 & 10,5 & & & & & & & 1 & 10,5 \\
\hline Calcaneus & & & 1 & 23,4 & & & & & 1 & 23,4 \\
\hline Mc III & 1 & 38,1 & & & & & & & 1 & 38,1 \\
\hline \multicolumn{11}{|l|}{ Mt II } \\
\hline Mt III & 3 & 81,9 & 2 & 26,6 & & & 1 & 61,6 & 6 & 170,1 \\
\hline Mt III+IV & 2 & 120,6 & & & & & & & 2 & 120,6 \\
\hline Mt IV & 1 & 5,5 & & & & & & & 1 & 5,5 \\
\hline Hauptstrahl-Mp unbestimmbar & 5 & 125,4 & 2 & 35,2 & & & & & 7 & 160,6 \\
\hline Nebenstrahl-Mp unbestimmbar & & & & & 1 & 1,1 & & & 1 & 1,1 \\
\hline \multicolumn{11}{|l|}{ Phal. 1 ant. } \\
\hline Phal. 1 ant./post. & 1 & 44,8 & & & & & & & 1 & 44,8 \\
\hline \multicolumn{11}{|l|}{ Phal. 2 ant. } \\
\hline Phal. 2 ant./post. & 1 & 22,6 & & & & & & & 1 & 22,6 \\
\hline Phal. 3 ant./post. & 1 & 26,1 & & & & & & & 1 & 26,1 \\
\hline Total Autopodium & 16 & 475,5 & 5 & 85,2 & 1 & 1,1 & 1 & 61,6 & 23 & 623,4 \\
\hline \multicolumn{11}{|l|}{ Plattenknochen indet. } \\
\hline Röhrenknochen indet. & 15 & 121,7 & 1 & 15,5 & & & & & 16 & 137,2 \\
\hline Gesamtergebnis & 97 & 4580,1 & 21 & 1941,9 & 2 & 40,5 & 15 & 1002,8 & 135 & 7565,3 \\
\hline
\end{tabular}

Tab. 20 Brugg-Kabelwerke 2007-2008 (Bru.007.3). Skelettteilspektrum Equiden in den Strukturen 1-2.

\begin{tabular}{lccc}
\hline & $\begin{array}{l}\text { Grösste Länge Faktoren (nach Koudelka 1885) } \\
(\mathbf{m m})\end{array}$ & WRH (cm) \\
\hline Humerus & 154,1 & 3,37 & 51,9 \\
\hline Radius & $151,8 / 149,2$ & 3,22 & $48,9 / 48,0$ \\
\hline Ulna & 177,7 & 2,67 & 47,3 \\
\hline Femur & $164,4 / 165,4$ & 3,01 & $49,5 / 49,8$ \\
\hline Tibia & $169,1 / 171,8$ & 2,92 & $49,4 / 50,2$ \\
\hline
\end{tabular}

Tab. 21 Brugg-Kabelwerke 2007-2008 (Bru.007.3). Masse und Wiederristhöhenberechnungen zum Hundeskelett FK 590, Pos. 1020. 


\begin{tabular}{|c|c|c|c|c|c|c|c|c|c|}
\hline \multicolumn{8}{|l|}{ Phase 2} & \multirow{2}{*}{\multicolumn{2}{|c|}{ Gesamtergebnis }} \\
\hline \multicolumn{2}{|l|}{ Grube 23} & \multicolumn{2}{|l|}{ Pfostenreihe } & \multicolumn{2}{|c|}{ Schichten bei cella } & \multicolumn{2}{|c|}{ Phase 2 Ergebnis } & & \\
\hline$n$ & g & $\mathrm{n}$ & g & $\mathrm{n}$ & g & $\mathrm{n}$ & g & $\mathrm{n}$ & g \\
\hline & & 2 & 8,2 & & & 2 & 8,2 & 4 & 30,6 \\
\hline & & 1 & 47,8 & & & 1 & 47,8 & 5 & 227,1 \\
\hline & & 5 & 36,7 & & & 5 & 36,7 & 15 & 1194,3 \\
\hline & & 6 & 112,5 & & & 6 & 112,5 & 11 & 282 \\
\hline & & & & & & & & 5 & 26,2 \\
\hline \multirow[t]{4}{*}{0} & 0 & 14 & 205,2 & 0 & 0 & 14 & 205,2 & 40 & 1760,2 \\
\hline & & & & & & & & 8 & 214,7 \\
\hline & & & & & & & & 1 & 10,6 \\
\hline & & & & & & & & 1 & 4,7 \\
\hline 1 & 11,4 & & & & & 1 & 11,4 & 6 & 43,5 \\
\hline \multirow[t]{5}{*}{1} & 11,4 & 0 & 0 & 0 & 0 & 1 & 11,4 & 16 & 273,5 \\
\hline & & & & & & & & 1 & 144,1 \\
\hline & & 1 & 303,2 & & & 1 & 303,2 & 17 & 1336,2 \\
\hline & & & & & & & & 6 & 494,8 \\
\hline & & & & & & & & 13 & 1651,3 \\
\hline \multirow[t]{5}{*}{0} & 0 & 1 & 303,2 & 0 & 0 & 1 & 303,2 & 37 & 3626,4 \\
\hline & & & & & & & & 4 & 75 \\
\hline & & & & & & & & 7 & 506,8 \\
\hline & & 1 & 397 & & & 1 & 397 & 4 & 1333,2 \\
\hline & & 1 & 35,5 & & & 1 & 35,5 & 6 & 181,9 \\
\hline \multirow[t]{9}{*}{0} & 0 & 2 & 432,5 & 0 & 0 & 2 & 432,5 & 21 & 2096,9 \\
\hline & & 6 & 62 & & & 6 & 62 & 7 & 72,5 \\
\hline & & & & & & & & 1 & 23,4 \\
\hline & & & & & & & & 1 & 38,1 \\
\hline & & 1 & 6,3 & & & 1 & 6,3 & 1 & 6,3 \\
\hline & & & & & & & & 6 & 170,1 \\
\hline & & & & & & & & 2 & 120,6 \\
\hline & & 1 & 6,7 & & & 1 & 6,7 & 2 & 12,2 \\
\hline & & & & & & & & 7 & 160,6 \\
\hline \multirow[t]{6}{*}{1} & 0,5 & 1 & 0,3 & 1 & 0,6 & 3 & 1,4 & 4 & 2,5 \\
\hline & & 1 & 57,4 & & & 1 & 57,4 & 1 & 57,4 \\
\hline & & & & & & & & 1 & 44,8 \\
\hline & & 1 & 24,3 & & & 1 & 24,3 & 1 & 24,3 \\
\hline & & & & & & & & 1 & 22,6 \\
\hline & & 2 & 38,3 & & & 2 & 38,3 & 3 & 64,4 \\
\hline \multirow[t]{3}{*}{1} & 0,5 & 13 & 195,3 & 1 & 0,6 & 15 & 196,4 & 38 & 819,8 \\
\hline & & 1 & 3,8 & & & 1 & 3,8 & 1 & 3,8 \\
\hline & & 1 & 2,9 & 1 & 18,4 & 2 & 21,3 & 18 & 158,5 \\
\hline 2 & 11,9 & 32 & 1142,9 & 2 & 19 & 36 & 1173,8 & 171 & 8739,1 \\
\hline
\end{tabular}

